\title{
Amino acids, independent of insulin, attenuate skeletal muscle autophagy in neonatal pigs during endotoxemia
}

\author{
Adriana Hernandez-García ${ }^{1}$, Rodrigo Manjarín' ${ }^{1}$ Agus Suryawan¹, Hanh V. Nguyen', Teresa A. Davis ${ }^{1}$ and Renán A. Orellana
}

\begin{abstract}
BACKGROUND: Sepsis induces loss of skeletal muscle mass by activating the ubiquitin proteasome (UPS) and autophagy systems. Although muscle protein synthesis in healthy neonatal piglets is responsive to amino acids (AA) stimulation, it is not known if AA can prevent the activation of muscle protein degradation induced by sepsis. We hypothesize that AA attenuate the sepsis-induced activation of UPS and autophagy in neonates.
\end{abstract}

METHODS: Newborn pigs were infused for $8 \mathrm{~h}$ with liposaccharide (LPS) (0 and $\left.10 \mu \mathrm{g} \cdot \mathrm{kg}^{-1} \cdot \mathrm{h}^{-1}\right)$, while circulating glucose and insulin were maintained at fasting levels; circulating AA were clamped at fasting or fed levels. Markers of protein degradation and AA transporters in longissimus dorsi (LD) were examined.

RESULTS: Fasting AA increased muscle microtubule-associated protein light 1 chain 3 II (LC3-II) abundance in LPS compared to control, while fed AA levels decreased LC3-II abundance in both LPS and controls. There was no effect of AA supplementation on activated protein kinase (AMP), forkhead box 01 and O4 phosphorylation, nor on sodium-coupled neutral AA transporter 2 and light chain AA transporter 1, muscle RING-finger protein-1 and muscle Atrophy F-Box/Atrogin-1 abundance.

CONCLUSION: These findings suggest that supplementation of AA antagonize autophagy signal activation in skeletal muscle of neonates during endotoxemia.

$S_{b}$ keletal muscle mass is maintained by a tight balance between protein synthesis, regulated by the mTOR pathway, and muscle degradation, regulated by the caspase-calpain, the ubiquitin-proteasome (UPS) and autophagy systems (1). During inflammation and sepsis, skeletal muscle protein synthesis is reduced and protein degradation is increased to deliver amino acids (AA) into the bloodstream in order to supply substrates to support the inflammatory response (2). It has been previously shown that mobilization of skeletal muscle AA leads to muscle mass loss, but minimizes overall body protein catabolism (3); however, the regulatory effect of free AA during inflammation and sepsis is not completely understood (4-6).

In healthy conditions, AA increase skeletal muscle protein synthesis, by stimulation of mTORC1 complex (7), and decrease protein degradation by limiting autophagy in the neonatal piglet (8). Briefly, during sepsis, there is an increase of plasma TNF- $\alpha$, interleukines and cytokines that will trigger the activation of skeletal muscle protein degradation (PD) (9). Activated protein kinase (AMPK) phosphorylation occurs in response to the intracellular starvation that take place with the catabolic state (10). This triggers the activation of a group of nuclear transcription factors known as forkhead box $\mathrm{O}$ (FOXO1 to 4), which increases the transcription of muscle RING-finger protein-1 (MuRF-1) and muscle Atrophy F-Box/ Atrogin-1 (atrogin-1) (10). The former two are main part of the UPS system, which is responsible for the control of protein degradation following myofibrillar breakdown $(11,12)$.

The autophagy-lysosome system plays a significant role in proteolysis. Microtubule-associated protein light 1 chain 3 (LC3) has been widely used as a marker of autophagy (13). Decreased levels of intracellular AA induce the formation of LC3II subunit, which parallels the development of the autophagosome $(13,14)$, and leads to activation of the autophagy-lysosomal degradation pathway (15). Under hypercatabolic conditions, formation of skeletal muscle autophagosomes contributes largely to lean mass loss associated to inflammation and sepsis $(16,17)$.

Neonatal growth is largely attributable to high rates of protein synthesis induced by the postprandial rise in insulin and AA $(18,19)$. Previous studies of our group have shown that AA transporters such as the light chain AA transporter 1 (LAT-1), the sodium-coupled neutral AA transporter 2 (SNAT-2) and the lysosome-associated membrane protein 2 (LAMP-2) play an important role on skeletal muscle protein synthesis activation by facilitating the AA entrance to the cell (20). Other studies in septic mice (21) have suggested that there is no alteration of these transporters during sepsis but less is known on this AA transporters adaptation during endotoxemia in the neonatal pig. Data generated from in vivo studies in neonatal pigs (7), rats $(22,23)$, and cell cultures $(24)$ indicate that the anabolic effect of AA on muscle protein synthesis is mediated through upregulation of the mTORC1 pathway, which controls both initiation and elongation phases of mRNA translation (25). Newborn-elevated rates of cell proliferation also lead to defects in newly synthesized proteins, activating autophagy pathways to regulate the degradation of cell components through the lysosomal machinery (26). We have previously shown that provision of AA to healthy neonatal pigs decreases autophagy in skeletal muscle (8), whereas AA administration augments muscle protein synthesis in 
endotoxemic animals (27). We now hypothesize that AA provision attenuates skeletal muscle protein degradation by decreasing UPS and autophagy system signal activation in an endotoxemic neonatal pig model induced by lipopolysaccharide (LPS). We also inquire if this AA effect on protein degradation pathway could be influenced by the availability of AA cellular transporters,

\section{RESULTS}

The increase in temperature and the metabolic response observed during the endotoxemic pancreatic-substrate clamps performed in neonatal pigs has been published (27); metabolic changes are briefly described in Table 1. Insulin levels in plasma increased in LPS and LPS plus supplemented AA (LPSAA) groups compared to control $(\mathrm{C})$ and control plus AA (CAA) $(P \leq 0.05)$. Plasma branched-chain amino acids (BCAA) levels were higher in AA supplemented groups vs. no AA-supplemented $(P \leq 0.05)$. Plasma glucose increased in healthy AA-supplemented group vs. controls $(\mathrm{C})$ only $(P \leq 0.05)$.

In Table 2, total AA concentration in the longissimus dorsi (LD) muscle was higher at fed compared to fasting AA levels for both $C$ and LPS animals $(P \leq 0.05)$, and tended to be higher in LPSAA compared to CAA. Likewise, histidine, leucine, valine, methionine, glutamine, serine, glycine, and cysteine were also higher in CAA and LPSAA compared to fasting counterparts $(P \leq 0.05)$. There were no differences between CAA and LPSAA for any of these AA. Compared to C and CAA, alanine was higher in LPS and LPSAA $(P \leq 0.05)$.

In Figure 1, AA-fed levels decreased the LC3-II-to-total LC3 ratio in both $\mathrm{C}$ and LPS groups $(P \leq 0.01)$. In Table 3, abundance of AA transporters, pro-caspase 3, $\alpha$-actin, phosphorylation of AMPK, FOXO1 and FOXO4, and abundance of MuRF-1 and Atrogin-1 did not differ between groups.

\section{DISCUSSION}

Elevated protein turnover rates during the neonatal period ensure adequate growth and remodeling in skeletal muscle $(10,18)$, making neonatal pigs highly anabolic even in the presence of an endotoxemic insult (28). We have previously shown that circulating plasma 3-methyl histidine, a marker of muscle protein degradation (29), is not increased in the presence of acute endotoxemia, especially when the catabolic endotoxemic

Table 1. Plasma concentrations of insulin $\left(\mu \mathrm{U} \cdot \mathrm{ml}^{-1}\right)$, glucose $\left(\mathrm{mg} \cdot \mathrm{dl}^{-1}\right)$, branched-chain amino acids $\left(\mathrm{nmol} \cdot \mathrm{ml}^{-1}\right)$, and urea nitrogen $\left(\mathrm{mg} \cdot \mathrm{ml}^{-1}\right)$ in neonatal pigs during an 8-h endotoxemic pancreaticsubstrate clamp

\begin{tabular}{lrrrr}
\hline Substrate & \multicolumn{1}{c}{ C } & \multicolumn{1}{c}{ CAA } & \multicolumn{1}{c}{ LPS } & LPSAA \\
\hline Insulin & $3.2^{*} \pm 0.5$ & $4.4^{\dagger} \pm 0.4$ & $6.6^{*} \pm 0.7$ & $8.9^{\dagger} \pm 0.8$ \\
Glucose & $66 \pm 4.0$ & $80^{* *,} \pm 5.0$ & $66 \pm 1.0$ & $61^{\dagger} \pm 2.0$ \\
BCAA & $542 \pm 39$ & $1,160^{* *} \pm 56$ & $404 \pm 51$ & $1,092^{* *} \pm 65$ \\
PUN & $25 \pm 3.0$ & $30^{\dagger} \pm 3.0$ & $24 \pm 2.0$ & $21^{\dagger} \pm 1.0$ \\
\hline
\end{tabular}

Amino acid concentration was targeted to fasting levels or fed levels while insulin and glucose were maintained at baseline fasting levels. Values are means $\pm \mathrm{SE}$; control (C), $n$ $=9 ;$ control + AA (CAA), $n=11 ;$ LPS, $n=6 ;$ LPS + AA (LPSAA), $n=8$.

BCAA, branched-chain amino acids; LPS, liposaccharide; PUN, plasma urea nitrogen. *Significantly different between control and LPS $(P \leq 0.05)$. * Significantly different

between fasting and fed $(P \leq 0.001)$. †Significantly different between CAA and LPSAA. response occurred during fasting (10). Since skeletal muscle protein turnover in healthy newborn piglets is high, allowing rapid growth through efficient protein deposition, we sustain that an adequate and constant intracellular AA availability is required, and for which autophagy pathways regulate and maintain the rate of degradation of muscle components through the lysosomal machinery. For this reason, we expected protein degradation markers to be elevated at baseline to a rank that may not be further augmented by LPS in the neonatal animal. Similarly, in the present study, LPS did not decrease skeletal muscle intracellular cleaved $\alpha$-actin, a marker of muscle degradation.

We have previously reported that AA, independent of insulin, regulate muscle protein synthesis in the neonate in both normal and endotoxemic conditions (27), but do not affect muscle protein degradation signaling (10). Similarly, phosphorylation of FOXOs, and AMPK, and abundance of

Table 2. Intracellular concentrations of free amino acids ( $\eta \mathrm{mol} /$ $\mathrm{ml}$ ) in longissimus dorsi muscle in LPS and control (C) pigs during endotoxemic pancreatic-substrate clamps

\begin{tabular}{|c|c|c|c|c|}
\hline AA & C & CAA & LPS & LPSAA \\
\hline \multicolumn{5}{|l|}{ EAA } \\
\hline Lys & $915 \pm 170$ & $1,135 \pm 142$ & $756 \pm 218$ & $1,202 \pm 193$ \\
\hline Arg & $365 \pm 92.5$ & $553 \pm 86.8$ & $388 \pm 115$ & $413 \pm 101$ \\
\hline His & $11.1 \pm 5.7$ & $24.3^{*} \pm 5.0$ & $15.3 \pm 6.83$ & $27.2^{*} \pm 6.4$ \\
\hline Thr & $119 \pm 18.4$ & $123 \pm 18.3$ & $91.8 \pm 18.5$ & $146 \pm 18.4$ \\
\hline Leu & $14.6 \pm 4.4$ & $39.5^{*} \pm 3.8$ & $17.3 \pm 5.3$ & $32.6^{*} \pm 5$ \\
\hline Ile & $55.9 \pm 9.4$ & $65.1 \pm 8.6$ & $77.4 \pm 10.9$ & $91.6^{*} \pm 9.9$ \\
\hline Val & $23.5 \pm 6.3$ & $56.5^{*} \pm 5.4$ & $28.4 \pm 7.7$ & $46.1^{*} \pm 7.6$ \\
\hline Met & $5.2 \pm 13.2$ & $46.9^{*} \pm 9.9$ & $8.28 \pm 15.1$ & $48.5^{*} \pm 12.6$ \\
\hline Phe & $155 \pm 16.3$ & $189^{*} \pm 16.2$ & $150 \pm 19.9$ & $189 \pm 18.9$ \\
\hline Trp & $10.9 \pm 2.85$ & $8.72 \pm 2.7$ & $11.9 \pm 2.9$ & $11.6 \pm 3$ \\
\hline \multicolumn{5}{|l|}{ NEAA } \\
\hline Asp & $55.8 \pm 15.9$ & $54.1 \pm 15.4$ & $51.1 \pm 16.8$ & $59.4 \pm 16.9$ \\
\hline Asn & $37.4 \pm 7.17$ & $38.6 \pm 6.65$ & $45.8 \pm 9.05$ & $44.5 \pm 9.2$ \\
\hline Glu & $257 \pm 51.8$ & $299 \pm 44.7$ & $273 \pm 59.5$ & $363 \pm 59.4$ \\
\hline Gln & $315 \pm 65.1$ & $413^{*} \pm 62.3$ & $424 \pm 72.7$ & $523^{*} \pm 73.3$ \\
\hline Ala & $187 \pm 32.3$ & $238 \pm 28.7$ & $314^{* *} \pm 36.3$ & $305^{* *} \pm 36.8$ \\
\hline Ser & $75.9 \pm 17.7$ & $158^{*} \pm 16.6$ & $76.3 \pm 25.3$ & $169^{*} \pm 24.9$ \\
\hline Gly & $416 \pm 98.6$ & $796^{*} \pm 83.4$ & $442 \pm 141$ & $874^{*} \pm 123$ \\
\hline Tyr & $18.3 \pm 4.7$ & $23.9 \pm 4.6$ & $17.9 \pm 5.31$ & $24.5 \pm 5.2$ \\
\hline Cys & $13.6 \pm 6.3$ & $40.5^{*} \pm 5.6$ & $6.4 \pm 7.42$ & $32.7^{*} \pm 7.1$ \\
\hline Pro & $57.3 \pm 18.4$ & $132^{*} \pm 14.6$ & $63.2 \pm 21.9$ & $86.2 \pm 21.8$ \\
\hline EAA & $1,101 \pm 229$ & $1,464 \pm 208$ & $887 \pm 252$ & $1,791^{*} \pm 268$ \\
\hline BCAA & $78.7 \pm 14$ & $139^{*} \pm 12.1$ & $105 \pm 15.9$ & $169^{*} \pm 17.1$ \\
\hline NEAA & $1,469 \pm 207$ & $2,296^{*} \pm 178$ & $1,803 \pm 235$ & $2,552 \pm 252$ \\
\hline TOTAL & $3,398 \pm 587$ & $4,543^{*} \pm 544$ & $3,915 \pm 638$ & $5,521^{*} \pm 673$ \\
\hline
\end{tabular}

Values are means \pm SE; control $(C), n=9 ;$ control $+\mathrm{AA}(\mathrm{CAA}), n=11 ; \mathrm{LPS}, n=6 ; \mathrm{LPS}+\mathrm{AA}$ (LPSAA), $n=8$.

*Significantly different between fasting and fed AA groups $(P \leq 0.05)$; ${ }^{* *}$ significantly different between $C$ and LPS, and between CAA and LPSAA $(P \leq 0.05)$.

AA, amino acids; LPS, liposaccharide. 


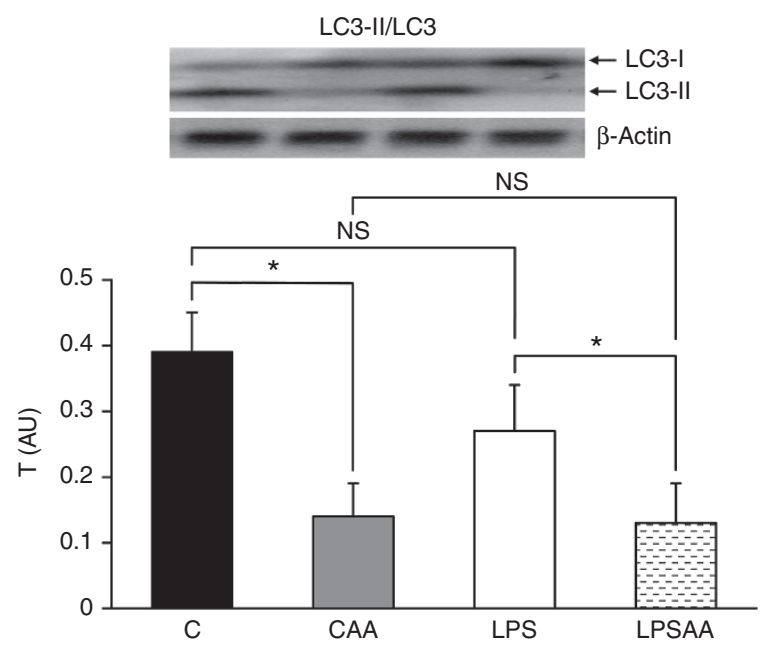

Figure 1. Effect of amino acid infusion on abundance of microtubuleassociated protein light 1 chain 3 (LC3-II/LC3Total; T); values are means \pm $\mathrm{SE}$; control (C), $n=9$; liposaccharide (LPS), $n=6$; control + AA (CAA), $n=11$; LPS + AA (LPSAA), $n=8$. *P $\leq 0.01$. AU, arbitrary units.

Table 3. Effects of AA on abundance and phosphorylation of protein degradation markers, in skeletal muscle of LPS and control pigs during endotoxemic pancreatic-substrate clamps

\begin{tabular}{|c|c|c|c|c|c|}
\hline Item (AU) & C & CAA & LPS & LPSAA & $P$ value \\
\hline AMPK (p) & $0.11 \pm 0.04$ & $0.12 \pm 0.03$ & $0.16 \pm 0.04$ & $0.13 \pm 0.04$ & 0.32 \\
\hline $\begin{array}{l}\text { Pro-caspase-3 } \\
(35 \mathrm{kda})\end{array}$ & $36.7 \pm 7.68$ & $30.4 \pm 6.41$ & $14.3 \pm 8.12$ & $20.5 \pm 7.21$ & 0.21 \\
\hline $\begin{array}{l}\alpha-\text { actin } \\
(14 \text { kda })\end{array}$ & $0.52 \pm 0.11$ & $0.30 \pm 0.09$ & $0.22 \pm 0.12$ & $0.22 \pm 0.10$ & 0.31 \\
\hline MuRF-1 & $3.76 \pm 1.71$ & $1.13 \pm 1.67$ & $1.86 \pm 1.73$ & $2.10 \pm 1.69$ & 0.59 \\
\hline Atrogin-1 & $5.50 \pm 1.40$ & $3.23 \pm 1.20$ & $4.68 \pm 1.46$ & $3.80 \pm 1.32$ & 0.60 \\
\hline LAMP-2 & $0.59 \pm 0.18$ & $0.48 \pm 0.15$ & $0.42 \pm 0.18$ & $0.39 \pm 0.17$ & 0.94 \\
\hline Fox0-1 (p) & $1.55 \pm 0.29$ & $0.69 \pm 0.23$ & $1.14 \pm 0.29$ & $1.12 \pm 0.27$ & 0.23 \\
\hline Fox0-4 (p) & $2.15 \pm 1.26$ & $2.65 \pm 1.08$ & $1.72 \pm 1.34$ & $1.61 \pm 1.25$ & 0.42 \\
\hline LAT-1 & $1.81 \pm 0.34$ & $2.03 \pm 0.34$ & $1.39 \pm 0.39$ & $1.22 \pm 0.36$ & 0.44 \\
\hline SNAT-2 & $1.61 \pm 0.36$ & $1.54 \pm 0.31$ & $1.20 \pm 0.38$ & $1.27 \pm 0.34$ & 0.68 \\
\hline \multicolumn{6}{|c|}{$\begin{array}{l}\text { Values are means } \pm \text { SE; control }(C), n=9 \text {; control }+\mathrm{AA}(\mathrm{CAA}), n=11 ; \mathrm{LPS}, n=6 ; \mathrm{LPS}+\mathrm{AA} \\
(\mathrm{LPSAA}), n=8 \text {. } \\
\text { Amounts of the phosphorylated forms ( } p \text { ) of each protein were normalized to its } \\
\text { abundance and estimated by western blot. } \\
\mathrm{AA} \text {, amino acids; LPS, liposaccharide; } \mathrm{AU} \text {, arbitrary units. }\end{array}$} \\
\hline
\end{tabular}

pro-caspase 3 and ubiquitin ligases remained unchanged in the present study. Interestingly, the LC3-II to total LC3 ratio decreased in response to AA supplementation in both control and LPS animals, indicative of an attenuation of the autophagic process under normal and endotoxemic conditions. LC3 is known to respond rapidly to catabolic conditions and decreased intracellular AA $(13,14)$, increasing the supply of substrates for energy and repair mechanisms. The finding that AA supplementation decreased autophagy markers in both groups suggests that endotoxemia does not inhibit the beneficial effects of AA supplementation on autophagy. Activation of the autophagy-lysosomal degradation by LPS may have provided AA for alternative energy production pathways or to supply substrate to maintain the systemic inflammatory response $(15,17)$.

In parallel to the changes observed in LC3, there was an increase in the total intramuscular AA concentration in both CAA and LPSAA groups. These results, in addition to the lack of response of LAT-1, SNAT-2, and LAMP-2 to LPS, suggests that AA transport into the muscle cells is unaffected by endotoxemia, as previously observed in mice (21). Noteworthy, BCAA intramuscular concentrations were higher in the LPSAA group compared to LPS alone and alanine was the only AA that increased in response to LPS, when compared to controls, and remained elevated after AA supplementation. This may indicate an increase in intracellular free AA flux in the presence of alterations in glucosealanine cycle that uses BCAA as an energy substrate in skeletal muscle in the presence of higher circulating AA concentrations (30). Taken together, these results suggest that AA, independent of insulin, regulate skeletal muscle autophagy in the neonatal pig in normal and endotoxemic conditions, which is associated with increased intracellular AA concentrations.

\section{METHODS}

\section{Animals and Experimental Design}

The protocol was approved by the Animal Care and Use Committee of Baylor College of Medicine and was conducted in accordance with the National Research Council's Guide for the Care and Use of Laboratory Animals. Thirty 1-2-d-old pigs from three different replicates were implanted with jugular vein and carotid artery catheters under sterile conditions. The catheters were flushed for patency with heparinized saline (100 IU ml ${ }^{-1}$; APP, Lake Zurich, IL) every $48 \mathrm{~h}$ and externalized and secured in the neck. The piglets continued with the sow for natural feeding until the day of the study. At $5-6 \mathrm{~d}$ of age $(2.05 \pm 0.34 \mathrm{~kg} \mathrm{BW})$, animals were randomly assigned to control $(n=20)$ or LPS $(n=14)$ groups. After $12-14 \mathrm{~h}$ of fasting, each animal was placed in a sling restraint system to perform endotoxemic pancreatic-substrate clamps for 8 consecutive hours, as previously described $(14,23)$. Briefly, insulin (200 mg.vial, Eli Lilly and Company, Indianapolis, IN) was infused at $7 \mathrm{ng} \mathrm{kg}^{-0.66} \mathrm{~min}^{-1}$ to achieve plasma concentrations of $2-5 \mu \mathrm{U} \mathrm{ml}^{-1}$ to simulate a fasting insulinemic state, while glucose was kept at fasting levels and circulating BCAA were maintained at target levels during the clamp by monitoring their concentrations every $5 \mathrm{~min}$ and adjusting the infusion rates of dextrose and a balanced AA mixture accordingly (27). The mixture of essential and nonessential AA contained (in mmol/l) 20.1 arginine, 12.9 histidine, 28.6 isoleucine, 34.3 leucine, 27.4 lysine, 10.1 methionine, 12.1 phenylalanine, 21.0 threonine, 4.4 tryptophan, 34.1 valine, 27.3 alanine (38\% provided as alanylglutamine), 12 aspartate, 6.2 cysteine, 23.8 glutamate, 17.1 glutamine (100\% provided as alanyl-glutamine), 54.3 glycine ( $4 \%$ provided as glycyl-tyrosine), 34.8 proline, 23.8 serine, 2.0 taurine, and 7.2 tyrosine ( $83 \%$ provided as glycyl-tyrosine) to supply requirements based on the composition of porcine body protein (18). After $6 \mathrm{~h}$ of continuous LPS infusion, the infusion rate of the balanced AA mixture was adjusted to clamp the plasma BCAA concentrations either to replicate fasting $(\sim 500 \eta \mathrm{mol} \mathrm{BCAA} / \mathrm{ml})$ or fed $(\sim 1,000 \eta \mathrm{mol} \mathrm{BCAA} / \mathrm{ml})$ levels and to maintain the plasma BCAA concentrations within $10 \%$ of the desired level (18). Animals were sacrificed after $8 \mathrm{~h}$ of receiving LPS, and $2 \mathrm{~h}$ after increasing the AA infusion rate to the fed level. Animals were euthanized with intravenous pentobarbital sodium $\left(50 \mathrm{mg} \mathrm{kg} \mathrm{BW}^{-1}\right)$.

\section{Muscle Degradation Signals and Markers}

Immediately following euthanasia, LD samples were stored at -70 ${ }^{\circ} \mathrm{F}$ until processing. Free intracellular AA concentrations in muscle were determined by high-performance liquid chromatography (PICO-TAG reverse-phase column; Waters, Mildford, MA,) using an analytical method based on deproteinization and derivatization of AA with phenylisothiocyanate, as previously described (18). 
AA transporter and intracellular markers of protein degradation and autophagy were measured by immunoblotting analysis, as previously described $(8,31)$. The antibodies used in the immunoblotting process were protein kinase B (total and Ser473, Cell Signaling Technology, Beverly, MA), AMPK- $\alpha$ (total and Thr172, Cell Signaling Technology), FOXO1 (total protein, Santa Cruz Technology, Santa Cruz, CA; Ser256, Cell Signaling Technology), FOXO4 (total protein and Ser262, Cell Signaling Technology), pro-caspase 3 (total protein, Cell Signaling Technology), MuRF1 (ECM Biosciences, Versailles, KY), atrogin-1 (ECM Biosciences), LAT1 (Cell Signaling Technology), SNAT2 (Aviva System Biology, San Diego, CA.), LC3 (Cell Signaling Technology), $\beta$-actin (Santa Cruz Biotechnology), $\alpha$-Actin (40 KDa; Dako-Cytomation, Glostrup, Denmark) and LAMP-2 (Cell signaling Technology, Danvers, MA)

\section{Statistical Analyses}

Data was analyzed by a two-way ANOVA using a linear mixed model in SAS 9.2 (PROC MIXED, SAS Institute, Cary, NC). The linear model included treatment as fixed effect, replicate as random effect, and initial BW as covariate. Normality of the residuals and presence of outliers were assessed by PROC UNIVARIATE (SAS 9.2), using the Shapiro-Wilk test, Q-Q-plots, and externally studentized residuals. Preplanned pairwise comparisons were analyzed using Student's $t$-tests. Data was presented as least square means \pm SE. Significant effects were considered at $P \leq 0.05$. The relationship between pro-caspase and $\alpha$-actin abundance in LD was analyzed using a correlation analysis in SAS (PROC CORR). Pearson correlation coefficient $(R)$ was considered significant at $P \leq 0.05$.

\section{ACKNOWLEDGEMENTS}

The authors thank Rosemarie Almonaci for technical support. This work is a publication of the U.S. Department of Agriculture/Agricultural Research Service Children's Nutrition Research Center, Department of Pediatrics, Baylor College of Medicine. The contents of this publication do not necessarily reflect the views or policies of the U.S. Department of Agriculture, nor does the mention of trade names, commercial products, or organizations imply endorsement by the U.S. Government.

\section{STATEMENT OF FINANCIAL SUPPORT}

This project has been funded in part by the National Institutes of Health awards, K08AR-51563 (R.A.O.), National Institute of Child Health and Human Development HD-072891 (T.A.D.) and R01 AR-44474 (T.A.D.), and the U.S. Department of Agriculture, Agricultural Research Service under Cooperative Agreement number 6250-51000-055 (T.A.D.).

Disclosures: The authors do not have any financial ties to products in the study or potential/perceived conflicts of interest to report.

\section{REFERENCES}

1. Lecker SH, Goldberg AL, Mitch WE. Protein degradation by the ubiquitin-proteasome pathway in normal and disease states. J Am Soc Nephrol 2006;17:1807-19.

2. Bruins MJ, Deutz NE, Soeters PB. Aspects of organ protein, amino acid and glucose metabolism in a porcine model of hypermetabolic sepsis. Clin Sci (Lond) 2003;104:127-41.

3. VerbruggenSC,Coss-BuJ,WuM, etal.Currentrecommendedparenteralprotein intakes do not support protein synthesis in critically ill septic, insulin-resistant adolescents with tight glucose control. Crit Care Med 2011;39:2518-25.

4. Orellana RA, Kimball SR, Nguyen HV, et al. Regulation of muscle protein synthesis in neonatal pigs during prolonged endotoxemia. Pediatr Res 2004;55:442-9.

5. Lang CH, Frost RA, Vary TC. Regulation of muscle protein synthesis during sepsis and inflammation. Am J Physiol Endocrinol Metab 2007;293:E453-9.

6. Joffe A, Anton N, Lequier L, et al. Nutritional support for critically ill children. Cochrane Database Syst Rev 2009;15;CD005144.

7. EscobarJ,FrankJW,Suryawan A, etal. Physiological rise in plasmaleucinestimulates muscle protein synthesis in neonatal pigs by enhancing translation initiation factor activation. Am J Physiol Endocrinol Metab 2005;288:E914-21.

8. Suryawan A, Davis TA. Regulation of protein degradation pathways by amino acids and insulin in skeletal muscle of neonatal pigs. J Anim Sci Biotechnol 2014;5:8.
9. Orellana RA, O'Connor PM, Nguyen HV, et al. Endotoxemia reduces skeletal muscle protein synthesis in neonates. Am J Physiol Endocrinol Metab 2002;283:E909-16.

10. Orellana RA, Suryawan A, Wilson FA, et al. Development aggravates the severity of skeletal muscle catabolism induced by endotoxemia in neonatal pigs. Am J Physiol Regul Integr Comp Physiol 2012;302:R682-90.

11. Jamart C, Gomes AV, Dewey S, Deldicque L, Raymackers JM, Francaux M. Regulation of ubiquitin-proteasome and autophagy pathways after acute LPS and epoxomicin administration in mice. BMC Musculoskelet Disord 2014;15:166.

12. von Haehling S, Genth-Zotz S, Anker SD, Volk HD. Cachexia: a therapeutic approach beyond cytokine antagonism. Int J Cardiol 2002;85:173-83.

13. Wang C, Wang Y, McNutt MA, Zhu WG. Autophagy process is associated with anti-neoplastic function. Acta Biochim Biophys Sin (Shanghai) 2011;43:425-32.

14. Kabeya Y, Mizushima N, Ueno T, et al. LC3, a mammalian homologue of yeast Apg8p, is localized in autophagosome membranes after processing. EMBO J 2000;19:5720-8.

15. Béchet D, Tassa A, Combaret L, Taillandier D, Attaix D. Regulation of skeletal muscle proteolysis by amino acids. J Ren Nutr 2005;15:18-22.

16. Mizushima N, Yamamoto A, Matsui M, Yoshimori T, Ohsumi Y. In vivo analysis of autophagy in response to nutrient starvation using transgenic mice expressing a fluorescent autophagosome marker. Mol Biol Cell 2004;15:1101-11.

17. Mofarrahi M, Sigala I, Guo Y, et al. Autophagy and skeletal muscles in sepsis. PLoS One 2012;7:e47265.

18. Davis TA, Fiorotto ML, Burrin DG, et al. Stimulation of protein synthesis by both insulin and amino acids is unique to skeletal muscle in neonatal pigs. Am J Physiol Endocrinol Metab 2002;282:E880-90.

19. Davis TA, Fiorotto ML. Regulation of muscle growth in neonates. Curr Opin Clin Nutr Metab Care 2009;12:78-85.

20. Suryawan A, Nguyen HV, Almonaci RD, Davis TA. Abundance of amino acid transporters involved in mTORC1 activation in skeletal muscle of neonatal pigs is developmentally regulated. Amino Acids 2013;45:523-30.

21. Laufenberg LJ, Pruznak AM, Navaratnarajah M, Lang CH. Sepsis-induced changes in amino acid transporters and leucine signaling via mTOR in skeletal muscle. Amino Acids 2014;46:2787-98.

22. Anthony JC, Yoshizawa F, Anthony TG, Vary TC, Jefferson LS, Kimball SR. Leucine stimulates translation initiation in skeletal muscle of postabsorptive rats via a rapamycin-sensitive pathway. J Nutr 2000;130: 2413-9.

23. Lynch CJ, Patson BJ, Anthony J, Vaval A, Jefferson LS, Vary TC. Leucine is a direct-acting nutrient signal that regulates protein synthesis in adipose tissue. Am J Physiol Endocrinol Metab 2002;283:E503-13.

24. Pham FH, Cole SM, Clerk A. Regulation of cardiac myocyte protein synthesis through phosphatidylinositol 3' kinase and protein kinase B. Adv Enzyme Regul 2001;41:73-86.

25. Kimball SR, Jefferson LS. Molecular mechanisms through which amino acids mediate signaling through the mammalian target of rapamycin. Curr Opin Clin Nutr Metab Care 2004;7:39-44.

26. Neel BA, Lin Y, Pessin JE. Skeletal muscle autophagy: a new metabolic regulator. Trends Endocrinol Metab 2013;24:635-43.

27. Orellana RA, Jeyapalan A, Escobar J, et al. Amino acids augment muscle protein synthesis in neonatal pigs during acute endotoxemia by stimulating mTOR-dependent translation initiation. Am J Physiol Endocrinol Metab 2007;293:E1416-25.

28. Orellana RA, Suryawan A, Kimball SR, et al. Insulin signaling in skeletal muscle and liver of neonatal pigs during endotoxemia. Pediatr Res 2008;64:505-10.

29. Rathmacher JA, Nissen SL. Development and application of a compartmental model of 3-methylhistidine metabolism in humans and domestic animals. Adv Exp Med Biol 1998;445:303-24.

30. Wolfe RR, Jahoor F, Hartl WH. Protein and amino acid metabolism after injury. Diabetes Metab Rev 1989;5:149-64.

31. Suryawan A, Nguyen HV, Bush JA, Davis TA. Developmental changes in the feeding-induced activation of the insulin-signaling pathway in neonatal pigs. Am J Physiol Endocrinol Metab 2001;281:E908-15. 